\title{
Crisis económica y crisis de la teoría económica. Notas para el debate
}

\author{
Alicia Puyana*
}

Perfiles Latinoamericanos, 26(51)

2018 | pp. 351-378

DOI: $10.18504 / \mathrm{pl} 2651-014-2018$

\begin{abstract}
Resumen
La crisis que enfrentó el mundo de la economía en 2007 intensificó el cuestionamiento del modelo neoclásico de oferta, sostén de las políticas económicas de las últimas tres décadas. Golpeó la economía real, la disputa política y la fundamentación teórica de la disciplina económica y de su enseńanza. Estos debates señalan la necesidad de revisar profundamente la teoría macroeconómica para rechazar los elementos fallidos y repensar cómo se forma y transmite el conocimiento en economía.
\end{abstract}

\begin{abstract}
The crisis facing the world economy since the end of 2007 has shaken to the core the economic paradigms that were the basis of models for economic policies and governmental roles for the last thirty years. It affected the real economy, the political dispute and the teaching of economics. So intensive debate illustrates the intensive need to deeply revise the macroeconomics theory, abandon the failed paradigms and construct new teaching curricula to produced professionals better equipped to analyze real word economic problems.
\end{abstract}

Palabras clave: economía, crisis económica, economía neoclásica, crecimiento económico, metáforas económicas, axiomas ergódicos.

Keywords: Economics, economic crisis, neoclassical economics, economic growth, economic metaphors, ergodic axioms.

* Doctora en Economía por la Universidad de Oxford (Reino Unido). Profesora investigadora, Flacso México | apuyana@flacso.edu.mx 


\section{Introducción}

L

a crisis que estallara a finales de 2007 ha sacudido los paradigmas teóricos que sustentaron las políticas económicas de los últimos treinta años. Las agencias multilaterales señalan mediocre y desigual recuperación de la economía mundial, de la Unión Europea, de Estados Unidos y de Japón, además de la desaceleración de China, Rusia y Brasil, entre otros (вм, 2016; Lagarde, 2015; FMI, 2014, 2015, 2016; Lane et al., 2014; OECD, 2016). Se abundó entonces en que el mundo tendría que lidiar con el estancamiento secular: largo periodo con bajas tasas de interés, pocas inversiones, productividad total factorial y laboral estancada, mínima inflación, alto desempleo y crecimiento disminuido e inestable (Summers, 2013, 2015), todo lo cual daña el ingreso y la equidad. El remesón al mercado de capitales de China y el derrumbe de los precios del petróleo y demás materias primas confirmaban estas previsiones y sus efectos en la estabilidad política, no solo en los países latinoamericanos.

La crisis fue un evento tan inesperado que la demanda de la reina Isabel de noviembre de 2007: "Es espantoso ¿Por qué nadie pudo prever tal desastre?" (RDMP, 2009) desató un debate entre economistas sobre las causas del choque, lo que las élites políticas y los medios de comunicación emularon. ${ }^{1}$ No surgieron respuestas definitivas, pero sí evidencias de las múltiples facetas de la crisis: económica, política, de la teoría económica, de la enseñanza de esta. Ni el mundo ha superado totalmente la crisis, ni las grandes instituciones financieras han logrado darle salida y mantener su poder (Blagrave et al., 2015; Davies, 2015). Sin respuestas definitivas, los planes de salida otorgaron a las empresas responsables, pero "demasiado grandes para quebrar", un generoso rescate financiero con recursos fiscales, aunque sin el requisito de modificar a fondo sus prácticas corporativas (Prins, 2015).

Sería inexacto sostener que nadie avizoró la crisis. No la vieron economistas neoclásicos ortodoxos como Lucas (2003), pero sí los de corte heterodoxo (Roubini \& Keen, 2006); si bien fueron ignorados por los portadores del pensamiento económico dominante y por los tomadores de decisiones. En efecto, en una práctica que Rogoff (2002), execonomista en jefe del FMI, denominó

1 Financial Times organizó un panel para debatir el futuro del capitalismo y mantiene el blog "El futuro del Capitalismo": http://blogs.ft.com/capitalismblog/. El presidente Sarkozy convocó a estadistas y científicos sociales a discutir "el Nuevo Mundo, Nuevo Capitalismo" y The Economist dedicó varios números a la crisis de la teoría macroeconómica y financiera, de lo que participaron, entre otros, el Premio Nobel Robert Lucas. 
represión neoclásica, ${ }^{2}$ durante años se han rechazado las posiciones que critican la ortodoxia (Galbraith, 2010; Gillies 2012). Estudios sobre el avance de la concentración de la riqueza y el ingreso, y sobre los riesgos para la estabilidad económica mundial de continuar el camino seguido desde los años ochenta, enriquecieron este debate con nuevos enfoques (Soros, 1998; Piketty, 2014).

Este ensayo se suma a los muchos que se han escrito acerca del impacto de la crisis de 2007 y de la necesidad de revisar los fundamentos teóricos de la economía y las políticas económicas. Repasa cómo anteriores crisis se transformaron en la oportunidad para reconsiderar los cánones establecidos y por qué algunas ideas sobreviven a su agotamiento debido a las características de la disciplina y a las medidas de apoyo a la investigación y difusión del conocimiento económico.

\section{Crisis económicas y crisis de la teoría económica}

Las respuestas a las preguntas sobre las razones de la crisis de 2007/2008 señalan dos procesos en continua retroalimentación: las transformaciones de la teoría económica desde el fin de la Segunda Guerra Mundial y la entronización de la escuela neoclásica, con la transición del análisis plural al reduccionista.

Las crisis económicas, como todas, obligan a repasar el rumbo de los acontecimientos, pues el desgaste epistemológico impulsa a resolver las limitaciones del paradigma central, debilita las fuerzas y los argumentos que sostienen el statu quo, y propicia transformaciones en las normas políticas y económicas (Alesina, Ardagna \& Trebbi, 2006). Otros sugieren que los paradigmas sobreviven más tiempo del debido, obligando a que la sociedad invierta recursos y tiempo para ajustar lo irreparable con arreglos marginales (Stigler, 1982a; Quiggin, 2010). Dan oxígeno de este modo a ideas zombi. Por la especificidad de la economía y de las ciencias sociales, ningún paradigma domina plenamente ni temporalmente en ellas como en las ciencias naturales (Kuhn, 1962). En las ciencias sociales coexisten, en competencia y con escaso diálogo, axiomas e interpretaciones del mundo contrarias. Algunas son más preeminentes y reciben mayor legitimidad. Según Kuhn, los elementos ideológicos innatos en las ciencias sociales dificultan el diálogo y el consenso deviene más de las relaciones de poder que de las propiedades de la investigación (Korinek, 2015).

2 "Más de un puñado de economistas internacionalistas guarda las cicatrices de no haber podido publicar, en los años de la 'represión neoclásica', artículos sobre la rigidez de los precios” (Rogoff, 2002: p. 9). Rogoff se refiere al rechazo de la tesis planteada por Dornbusch en 1975 sobre los precios y los mercados rígidos que limitan el ajuste automático de los mercados a la situación de equilibrio, en contradicción a los planteamientos de Lucas. El aporte de Dornbusch es considerado un avance significativo de la economía neoclásica, lo que desplazó a Lucas como texto de macroeconomía.

A. Puyana | Crisis económica y crisis de la teoría económica. Notas para el debate Perfiles Latinoamericanos, 26(51) | FLACso México | pp. 351-378 | DoI: 10.18504/pl2651-014-2018 
En la economía moderna, tres grandes eventos marcaron el fin de tres etapas del pensamiento económico y revelaron la mutabilidad de los paradigmas.

El primero, la Gran Depresión de los años treinta, significó la conclusión de una etapa de acelerado crecimiento del producto, el comercio global y avances tecnológicos; el segundo, la estanflación de mediados de los años setenta, que originó la crisis de la deuda y puso fin a la edad de oro del capitalismo; el tercero, el choque de los mercados de valores de 2008, raíz de la Gran Recesión, del golpe de gracia para la Gran Moderación, y del periodo (inicios de los ochenta-mediados de 2007) de baja inflación y crecimiento sostenido —si se ignoran las caídas del producto en México, Argentina, Turquía y el Sudeste Asiático, entre 1982 y 2002.

\section{La Gran Depresión}

La Gran Depresión minó la certeza de que el mercado tenía la capacidad de realizar los ajustes necesarios para superar los ciclos. El colapso del mercado de valores en 1929, el estancamiento económico, las caídas de la demanda y las ganancias revelaron que se debía intervenir para tratar las imperfecciones del mercado y mantener la economía. Al develar las limitaciones de los mecanismos del mercado, la crisis de 1929 mostró la incapacidad de los instrumentos monetarios para reactivar la economía. Ante esta evidencia, tomaron fuerza, por una parte, la demanda efectiva, como catalizador del crecimiento y, por la otra, las políticas para estimularla y sostenerla en épocas de crisis.

Para Keynes las crisis no son episodios casuales, por lo que el buen funcionamiento del capitalismo requiere de una agencia, un papel que le corresponde al Estado que, cuando las circunstancias lo exigen, invierte para mantener el empleo y sostener la demanda. Keynes criticó también al sector financiero por su propensión a la especulación cortoplacista.

Relevante es el rechazo de dicho autor al método ergódico de la teoría económica clásica cuando plantea que los axiomas ergódicos y sus resultados son aplicables solo a casos específicos, y utilizarlos universalmente en situaciones distintas generarían resultados adversos (Davidson, 2012: p. 3). ${ }^{3}$ No faltaron economistas que, aplicando la parábola de los vidrios rotos, vieran en la crisis una gran oportunidad, enfatizaran los efectos constructivos de la destrucción y abstrajeran sus costos económicos y sociales para subrayar que toda acción anticíclica causaría mayor daño que la crisis. Hoy se calla que, al retiro prematuro

3 Keynes rechazó la neutralidad del dinero y que este y los bienes de capital sean sustitutos. 
del New Deal, la economía estadounidense volvió a declinar y solo se recuperó con el gasto militar de la Segunda Guerra (Krugman, 2009b).

\section{El final de la edad de oro}

Keynes dominó el pensamiento económico y la acción política desde el fin de la guerra hasta mediar los años setenta. Durante esta etapa, la edad de oro del capitalismo (Scott, 1991), todos los países crecieron a tasas elevadas, con bajos desempleo e inflación. En el tiempo, la acelerada expansión presionó las fuentes de recursos naturales, indujo inflación e instaló su agotamiento en el panorama intelectual y político, con el Club de Roma y la opep.

El análisis de la edad de oro del capitalismo y su terminación ha de considerar el entorno político de la Gran Depresión, la segunda posguerra, el desafío de la en ese entonces Unión Soviética y el afianzamiento de los Estados Unidos, que justificaron el estímulo a la demanda interna por la vía del empleo, los salarios y los ingresos, contenidos en un Estado de bienestar. El modelo se agotó ante la imposibilidad de mantener, simultáneamente, altas inversiones, elevado gasto público, pleno empleo, ganancias crecientes y control de la inflación, con demandas salariales elevadas y estancamiento de la productividad. Contribuyó además el debilitamiento de la cooperación internacional y la salida de los Estados Unidos, en 1971, de la libre convertibilidad del dólar, por la crisis de liquidez de su economía. Las tasas de cambio flexible reemplazaron las fijas, dispararon la inflación, fortalecieron las expectativas racionales, la neutralidad del dinero y el rechazo del uso de políticas fiscales para manejar la producción, el empleo y el ingreso. Este avance del monetarismo resultó de la incapacidad de la economía keynesiana de explicar la "estanflación" de inicios de los años setenta que desacreditó las políticas anticíclicas y, al atribuir la crisis y la inflación al estímulo de la demanda vía gasto público, deslegitimó las políticas activas de empleo (Lucas \& Sargent, 1979) y retomó elementos de la teoría económica clásica, centrados en la estabilidad de precios y del producto. A la síntesis neoclásica, los neokeynesianos sumaron su búsqueda de fundamentos microeconómicos para el análisis macroeconómico, pero dejaron sin resolver los problemas de agregación y de identificación (Marchionatti \& Sella, 2015).

Cobraron entonces fuerza los modelos dinámicos de equilibrio general ${ }^{4}$ y sus supuestos de ergodicidad, racionalidad individual y eficiencia de los mercados;

4 Para un pormenorizado análisis de las restricciones y de los aportes que los modelos de equilibrio general —en particular los de equilibrio general dinámico estocástico (EGDE) — han dado al estudio de la macroeconomía, véase Korinek (2015). Estos modelos dominan el análisis económico en las universi-

A. Puyana | Crisis económica y crisis de la teoría económica. Notas para el debate Perfiles Latinoamericanos, 26(51) | FLACso México | pp. 351-378 | DoI: 10.18504/pl2651-014-2018 
fueron incorporados plenamente en modelos econométricos con el individuo representativo como actor principal. Por los supuestos de racionalidad perfecta e información completa, la política económica se tildó de ineficaz para disminuir el desempleo ya que los individuos conocen el mercado, anticipan que el aumento del gasto público produce inflación, ajustan precios y salarios, e impiden el aumento del desempleo y la corrección salarial, incluso de corto plazo (Kaletsky, 2009). En estos modelos no cabe el desempleo y la única forma de incluirlo fue considerándolo decisión voluntaria de individuos racionales e informados, como en Lucas \& Sargent (1979). A la inflación de los años setenta y a la crisis de la deuda al despuntar los ochenta, siguieron los ajustes fiscales, la liberalización económica y las reformas estructurales, que no fueron suficientemente estructurales, pues eliminaron las restricciones al mercado provenientes de las acciones del Estado y mantuvieron intacta la supresión de los intercambios del sector privado que estaban ligados a la concentración de los capitales productivo, financiero, comercial y del conocimiento (Lipton, 1992).

\section{La gran moderación, o los peligros de la estabilidad}

La liberalización de la economía y el retiro del Estado del manejo económico, ${ }^{5}$ en respuesta a la crisis de la deuda y la inflación, marcó las rutas de la teoría y la política económicas y los fundamentos de la organización social. Entronizó en la teoría y la política macroeconómicas los axiomas ergódicos neoclásicos y encumbró al mercado y al individualismo a rectores de la acción social. La práctica económica y la política se centraron en la ideología liberal: "La sociedad no existe, hay individuos, hombres y mujeres y familias", resumía Margaret Thatcher (1987: p. 1. Traducción propia).

Lejos de ser un proyecto estrictamente técnico que afectara solo la economía y buscara exclusivamente la eficiencia del gasto público, las reformas trastocaron la estructura del poder político y la distribución del excedente económico, transformando las relaciones entre el Estado y la sociedad y entre el capital y el trabajo. Al redefinir las fronteras del Estado erigió la rentabilidad del capital como el eje de la economía y la eficiencia, la rentabilidad y la competitividad de las normas de las políticas públicas, relegando la equidad a plano secundario. Esta lógica consagró la separación entre ciencia económica positiva y normativa, ignorando que eficiencia y equidad forman una unidad y "[...] como tal deben

dad y en los centros de decisión política: FMI, BM, OCDE, y en las bancas centrales de casi todo el mundo (Francesco, 2015).

5 Bases y antecedentes de estas políticas liberales se encuentran en Hayek (1990). 
ser tema de la economía política y objeto de discusión de las democracias y no, como hoy, centrar el debate en si con democracia el mercado asegura o no la eficiencia paretiana" (Stiglitz, 1991: p. 43. Traducción propia). Se consolidó de este modo la distinción entre las políticas económicas y las políticas sociales y la equidad se aisló de las primeras. Con esta óptica, hubo mayor tolerancia a los niveles de pobreza, desigualdad, exclusión y desempleo, concebidos antes como moralmente inaceptables.

Aislar la política social de la económica lleva a la falsa pregunta de si hay objetivos sociales y objetivos económicos independientes y contradictorios, $y$ al definir el crecimiento como el objetivo final de toda política económica se subsume a este todas las demás (Lynn, 2003: p. 129). De instrumento de desarrollo, el crecimiento deviene en fin: "Concebimos el desarrollo social como el complemento natural del desarrollo económico, por su valor intrínseco e instrumental" (вм, 2005: p. 2. Traducción propia). La política social, reducida a complemento de la económica, ni busca ni puede afectar su naturaleza. Los programas de pobreza se limitan a aliviar los efectos más severos de la pobreza extrema, pues pueden conducir al "desencanto" con la democracia o con la globalización y generar conflictos sociales de resultados imprevisibles.

Con el modelo neoliberal entró La Gran Moderación, nombre acuñado por James Stock (2002), legitimado por Ben Bernanke y que hace referencia a la estabilidad de precios y del producto. Se ha blandido como confirmación empírica del éxito del liberalismo y del poder del mercado para definir la óptima ubicación de factores. Esta enunciación ignora las crisis de Estados Unidos de los ochenta, las mexicanas $(1986,1994,2009)$ y las del Sudeste Asiático, Turquía, Colombia y Argentina en los noventa. El éxito de la moderación reside en la selección de casos no en sus méritos.

Bernanke (2004) consideró como causa principal de la Gran Moderación la restricción monetaria y la independencia de los bancos centrales y desechó los motivos de carácter político y estructural: dinero fácil, mercados desregulados, monedas revaluadas, importaciones baratas, menores choques externos. La Gran Moderación dio pábulo a que Lucas declarara resuelto el manejo de los ciclos económicos y redujera el papel de la macroeconomía a definir los estímulos para inducir a los individuos a trabajar y ahorrar: bajos impuestos y gasto público moderado, convencido de que los beneficios en bienestar de las mejores políticas fiscales de oferta de largo plazo superan en mucho los beneficios potenciales del manejo de corto plazo de la demanda, por óptimo que sea (Lucas, 2003).

Mercados eficientes y precios correctos fueron los paradigmas teóricos que avalaron la desregulación de los mercados financieros y de bienes, y orientaron las privatizaciones y las fusiones de empresas. Todo parecía marchar hasta que el estallido de la burbuja inmobiliaria en 2007-2008 enterró la Gran 
Moderación. Varios economistas, usando estadísticas simples pero reveladoras (Galbraith, 2010), llamaron la atención sobre los peligros de esos axiomas. Minsky (2008) había advertido que largos periodos de estabilidad inducen a tomar mayores riesgos y a tasas de rentabilidad superiores, los cuales devienen en sistemas Ponzi (como Madoff). Las ideas de Minsky contradecían las afirmaciones de Greenspan: "Las tecnologías de la información han expandido tanto los mercados que los gobiernos, aun los incrédulos, no tienen otra alternativa que desregular [...] Sin duda, hoy los mercados financieros mundiales son más eficientes que nunca" (Greenspan, 1998: p. 1). La crisis fue prevista y era evitable, solo la indiferencia o la ligereza lo impidieron, concluyó la Comisión para Investigar la Crisis Financiera (FCIC, siglas en inglés) del Congreso de los Estados Unidos (FCIC, 2011).

La crisis iniciada al finalizar 2007 ha sido larga para los estándares de la segunda posguerra y la recuperación lenta y evasiva, a la luz de la turbulencia de los mercados de capitales por el colapso en China a inicios de enero 2016. Tampoco es claro cuáles axiomas serán sustituidos y la resistencia al cambio es poderosa. En comparecencia ante el Congreso estadounidense para explicar la crisis financiera, Greenspan declaró en 2008 que habian colapsado las bases intelectuales (las hipótesis de los mercados eficientes y la de los precios correctos) sobre las cuales se habian edificado las politicas macroeconómicas de la Gran Moderación, pues los modelos no valoraron el riesgo (Wall Street Journal, 2008). ${ }^{6}$ En el mismo foro, Stiglitz (2009) señaló las causas y actores responsables de la crisis: los bancos, los fondos financieros, las agencias controladoras y los gobiernos, al incumplir su deber de proteger a los ciudadanos.

\section{Escuelas de pensamiento y visión dominante}

Tras casi nueve años del estallido de la crisis, continúa el debate sobre sus raíces y alcances (Gambina, 2010; Coyle, 2012a) y, desde la economía y otras disciplinas, ganan fuerza las críticas a las corrientes económicas ortodoxas que al responder "por qué no advirtieron la crisis" añaden: ¿cómo llegamos allí y cómo salir del estancamiento?, con lo cual abren compuertas a cuestionar las razones por las cuales no aparece aún la esperada transformación teórica $(\mathrm{Gi}-$ llies, 2012; Coyle, 2012a; Rosenthal, 2010). Los eventos y las políticas vigentes reflejan el endurecimiento de posiciones ortodoxas que han introducido

6 Pero dieciocho meses más tarde, si bien señaló como causa inmediata de la crisis la "proliferación global de los títulos tóxicos de crédito", exoneró de toda culpa su política de dinero barato y gran desregulación (Wall Street Journal, 2008). 
el nuevo consenso monetario con la estabilización de precios y la maximización de las ganancias financieras, sus objetivos centrales. Una explicación, si bien parcial, señala el carácter de la teoría macroeconómica, ligada al poder económico y político y a la estructura de su comunidad: pequeña, cerrada a los iniciados, estructurada oligárquicamente, con un sistema para financiar la investigación que, en los cincuenta últimos años, ha privilegiado estudios de corte neoclásico, en modelos dinámicos estocásticos de equilibrio general dinámico (Collander, 2010; Syll, 2013).

\section{La visión dominante}

A diferencia de las ciencias naturales, en la economía conviven escuelas de pensamiento con supuestos y propuestas diferentes y contrarios - marxistas, institucionalistas, poskeynesianos, austríacos, neorricardianos, evolucionistas, sraffianos, por mencionar solo las más relevantes (Gillies, 2012)—. Esta convivencia deviene de la naturaleza de la disciplina, en la cual, a pesar de las divergencias ontológicas, epistemológicas y metodológicas, las escuelas se confrontan y debaten tópicos específicos con lo que renuevan la disciplina y mejoran su comprensión de la realidad (Garnett, 2011; Mearman, 2011). No obstante, el debate no entraña procesos de selección de la mejor teoría, donde gana la idea más sólida. Por ello, la corriente dominante en economía debe entenderse en el contexto político que reine a escala mundial y no como un desarrollo desde la academia. Así, en 1947, intelectuales, empresarios y políticos fundaron la Sociedad de Mont Perelin para impulsar nuevas interpretaciones de eventos económicos y políticos, como la crisis de los años setenta y la caída de las instituciones de Bretton Woods (Udry, 1997) y abrieron el camino a la corriente, genéricamente denominada neoliberalismo, la que se acopló bastante bien a las reformas estructurales promovidas desde el centro (Harvey, 2007) que, en algunas latitudes (el Chile de Pinochet o la Argentina de Videla), se instauró bajo regímenes dictatoriales.

El problema de la teoría neoclásica no es erigir el mercado como el óptimo asignador de recursos, ni recurrir a la modelización matemática, sino sostener los axiomas ergódicos como sus basamentos epistemológicos y convertir el instrumento - la matematización, en sí neutra - en el centro del conocimiento; asumir que la realidad se puede estilizar en regularidades predecibles, determinísticas o estocásticas, para sustentar que el análisis económico debe formalizarse en modelos matemáticos (Korinek, 2015). Del énfasis en una herramienta se pasa a un supuesto ontológico sobre la realidad social, como sistema cerrado y regular que encierra supuestos contradictorios como el atomismo y la independencia y 
racionalidad de los agentes. Los supuestos de la escuela neoclásica varían significativamente y conviven aunque comporten contradicciones y hallazgos empíricos opuestos. Las interpretaciones no ortodoxas de la crisis por economistas de este sello son variaciones del canon neoclásico estándar (Farrell \& Quiggin, 2012), como lo es la disputa sobre los alcances del mercado para satisfacer las necesidades sociales que sostuvieron los economistas de "agua dulce" (Friedman, Lucas, Sargent, Barro o Fama) con los de "agua salada" (Krugman, Stiglitz, Blanchard, Mankiw o Strauss-Kahn). Por ejemplo, vertiente neoclásica, la economía conductual (behavioral economics) vincula la economía neoclásica con la psicología; asume que el actor racional enfatiza el presente; tiene aversión a las pérdidas y exagerados optimismo y pesimismo constituyen el paso más reciente en la individualización de la macroeconomía; niega que a más invisible la mano invisible y más libre el mercado, mayores tiendan a ser la manipulación y el engaño y, por lo tanto, superior la necesidad de control público (Akerlof $\&$ Shiller, 2015).

A partir de Smith y Ricardo, la teoría económica liberal se basa en metáforas de la física (Gillies, 2012; Jomo, 2005), en las cuales la sociedad, como el universo, se rige por fuerzas naturales que mantienen el equilibrio del cosmos y lo restauran luego de cataclismos. Las fases de la luna, los eclipses, los monzones, se repiten y ninguna acción del hombre puede evitarlos, así pueda predecirlos con cierta precisión. En la economía, y en la sociedad, la mano invisible conserva el equilibrio y al romperse lo restaura, a menor costo al que resultaría de intervenir manos visibles.

Samuelson echó los fundamentos matemáticos necesarios para elevar la economía del reino de la historia al de la ciencia (Samuelson, 1970). ${ }^{7}$ Adoptar el método de las ciencias naturales implicaba que la teoría económica debía construir axiomas ergódicos aceptando que el futuro económico está predeterminado por procesos estocásticos (Samuelson 1969). Ya que los eventos económicos se repiten inexorablemente en trayectoria conocible, el único oficio de los economistas es calcular las distribuciones probabilísticas de los precios y del producto futuros pues, a partir de los hechos pasados y, sin contemplar las condiciones iniciales, es factible predecirlos y responderles, sin alterar su curso: "[...] los actores económicos, movidos por su interés individual y con información confiable sobre el futuro, invertirán correctamente en las actividades que den los retornos más elevados y garantizarán la prosperidad global" (Davidson, 2012: p. 3. Traducción propia). Con variaciones importantes, en tales axiomas

$7 \quad$ El mayor logro de Samuelson como economista fue precisamente hacer de la economía una ciencia cuando introdujo en ella los métodos de las ciencias naturales. Así lo expuso en una entrevista en 1970 (Samuelson, 1970). 
se basaron muchos autores (Lucas y Sargent, Cochrane, Stiglitz, Mankiw, M. Friedman, Scholes), quienes consideraron este método como el único que satisface los requisitos científicos apropiados para la investigación económica y base racional de la política pública.

Aplicar en la economía los métodos de las ciencias naturales fue la respuesta para satisfacer la necesidad de seguridad y certidumbre de los espiritus animales, sostén del capitalismo. "Elevar" la economía al rango de las ciencias naturales cubrió a ciertos economistas con neutralidad política, el nuevo traje del emperador, convirtiendo sus propuestas en axiomas irrefutables, más allá de todo contexto social, político, histórico.

En los años setenta, la ciencia económica se sumergió en el proyecto de asimilar la macroeconomía a la microeconomía, buscando como única explicación científica de los fenómenos de la primera los comportamientos de los individuos de la segunda. Así, los economistas, armados con las metáforas y algunas herramientas de la física y con el arsenal de largas series de tiempo para universos amplios, decenas de países, múltiples variables, maquinas poderosas y sofisticado software, buscaron, como los físicos, una ley universal que lo explicara todo. Pero "[...] sólo una teoría podía explicarlo todo: la basada en información completa, la racionalidad del agente representativo y la eficiencia del mercado. Cualquiera otra tendría que integrar factores que sepultarían el axioma de la libre competencia en mercados perfectos: las imperfecciones del mercado, las economías de escala, las fallas de la información y la irracionalidad de los actores" (Kay, 2014).

La teoría macroeconómica abandonó el complejo mundo real y se alejó de los senderos explorados por economistas clásicos y por pioneros de la economía del desarrollo, que enfatizaron los retornos crecientes a escala o el papel de la historia y de las instituciones, difícilmente modelables en ese entonces (Krugman, 1999). La economía ortodoxa evolucionó en el marco de la competencia perfecta, los retornos decrecientes, tomó como realidad la simplificación que se podía modelar y retrocedió a la "edad media de la macroeconomía" al desechar los avances de los años treinta y cuarenta (Krugman, 2009a, 2011). En aras de la elegancia, sacrificó el método científico de comprobar las hipótesis con la realidad y, poco a poco, el economista se preocupó más por tener impacto y menos en investigar (Fullbrook, 2013; Solow, 2010; Stigler, 1982a). Esta metamorfosis, apuntó Galbraith (1974: pp. 239-240), al posesionarse como presidente de la American Economics Association, no respondía únicamente a la necesidad de manejar el riesgo, sino a la pretensión de subordinar el Estado, y la sociedad, a los dictámenes del mercado y a "[...] rechazar toda herejía, en cualquier forma organizada, es decir, cualquier cosa que pareciese amenazar la santidad de la propiedad, los beneficios, la política arancelaria adecuada, el presupuesto 
equilibrado, o implicase simpatía hacia los sindicatos, la propiedad pública, la regulación pública, o los pobres”. Añadía que, en el proceso de excluir el poder del análisis económico, "[...] y convertir la teoría económica en una disciplina no política, la teoría neoclásica destruyó su relación con el mundo real”.

El énfasis matemático propone un modelo lógico deductivo, en el cual las características del sistema se establecen no en función de la realidad externa sino de la consistencia interna del modelo, basado en equilibrio estático y lineal en el que la oferta y la demanda se igualan a un precio dado y las propiedades macroeconómicas son la suma de las microeconómicas (Blanchard, 2010; Silim, 2012; Fullbrook, 2013). ${ }^{8}$ Por lo cual se forman economistas que, en cargos en el sector privado o en el público, tienen dificultades para interpretar las realidades concretas a partir de la empírea (Coyle, 2013; Madi et al., 2015); no comprenden las preguntas ni las propuestas de otras escuelas y, por falta de alternativas aprendidas, reproducen el statu quo. Para la mayoría de los economistas que no emprende carrera académica, los años de formación en el pregrado son clave, pues es la visión que aplicarán en sus prácticas profesionales (Coyle, 2012a). Este arsenal matemático, unido a una escasa formación en otras escuelas de pensamiento, afectó también la relación de la teoría económica con las demás ciencias sociales. Todo lo cual limita la comprensión de los problemas reales e impide comprender las limitaciones de la aplicación de los modelos neoclásicos a las políticas. La supuesta superioridad científica de la teoría económica (Becker, 1996) impulsó una suerte de "imperialismo de la economía sobre las ciencias sociales", que fomentó entre las demás disciplinas la adopción acrítica de los axiomas económicos ergódicos hoy cuestionados. Los mercados perfectos y las expectativas racionales invadieron el estudio sobre la racionalidad del electorado, de las instituciones y de la historia económica. Es también evidente que los programas sociales reemplazan los derechos sociales del Estado de bienestar con programas de transferencias focalizadas. En estos se asume que los pobres son seres racionales y que como tales responderán correctamente a los estímulos y sanciones burocráticamente establecidos. Una vez dotados de educación y salud básicas, los niños de hogares en pobreza extrema, serán individuos dotados de las habilidades y el conocimiento suficientes para competir y triunfar en mercados supuestamente perfectos, en sociedades imaginariamente meritocráticas, que retribuyen el esfuerzo individual. La sociedad ha cumplido su compromiso, la responsabilidad es del individuo. El mercado resolverá el problema del calentamiento global y la contaminación.

8 Estos modelos tienen fallas epistemológicas al estructurarse con falacias, ya las de composición, ya de peticiones de principio. 
Al mediar 2016, cabe preguntar en qué grado la crisis ha puesto en discusión las ideas económicas dominantes, o si mantienen su vigencia entre académicos, políticos y administradores públicos, no obstante su poca capacidad explicativa y erróneas predicciones (Quiggin, 2010). Indican lo segundo, el tratamiento de la crisis financiera del euro por la insolvencia de Grecia y la reacción de prestigiados economistas al Brexit que receta aplicar las leyes y normas comunitarias, impedir el sobreendeudamiento de gobiernos y bancos e intensificar las medidas de austeridad (vOX-CEPR, 2016).

Presentamos las principales ideas de la escuela neoclásica, en escrutinio a raíz de la crisis de 2007. El tiempo dirá si prevalecerán las que pasen la prueba de los eventos o las que velen por la elegancia y parsimonia de los modelos. Greenspan (2013) reitera la vigencia de los axiomas ergódicos de los modelos de equilibrio general (MDEG) y reconoce que el ser humano no es siempre irracional, lo que convertiría los pronósticos en un cara-y-sello. Afirma que la irracionalidad se repite sistemáticamente, por lo que es modelable con supuestos de la economía del comportamiento. Los modelos de equilibrio general deben " $[. .$.$] incorporar ecuaciones que, en lo posible, midan y pronostiquen$ el comportamiento humano y la cultura corporativa [...] así podemos mejorar nuestro pobre desempeño de los últimos cinco años" (Greenspan, 2013. Citado en Frank, 2015).

Quizás la primera idea en cuestión sea la supuesta información completa sobre productos, precios y riesgos. La crisis hipotecaria y de las burbujas especulativas no hubiera ocurrido si todos los inversionistas fueran racionales y hubieran conocido el valor exacto de todas las opciones de inversiones. Serían seres con superpoderes para discernir entre los cientos de miles de datos sobre precios y utilidades y decidir óptimamente (Foyle, 2004). La exuberancia de datos y su manejo no impidió que la crisis llegara y estallara. Tratándose de agentes financieros de elevadísima sofisticación, resulta dudoso que se trate de un problema de racionalidad: o la información disponible es imposible de procesar y por lo tanto incompleta, o la racionalidad implica características diferentes a las propuestas teóricas, como la tendencia al engaño (Akerlofy \& Shiller, 2015).

Otro axioma en crisis es la tasa natural de desempleo que, pese a su imposible demostración, ha guiado la política económica y legitimado alto desempleo y empleo precario e informal, inducidos por las estrategias centradas en el crecimiento del pIB con baja inflación, evidentes en las declaraciones de la jefa del Banco de la Reserva al elevar las tasas de interés, en diciembre de 2015 (Yellen, 2015). Hoy se duda el retorno automático del mercado laboral al equilibrio en pleno empleo y propone aceptar, como normales, una recuperación sin empleo 
(jobless recovery) y el crecimiento sin empleo (jobless growth) (Farmer, 2009). Los crecientes desempleo y empleo precario y la mermada participación laboral, ponen en duda cuál es el límite de la tasa "natural" que conduce al debilitamiento de la demanda interna y a riesgos de crisis políticas y económicas.

Enfoques más globales señalan la hipótesis de los mercados eficientes (HмЕ). ${ }^{9}$ Hipótesis basada en la racionalidad de los inversionistas y los hogares, los que al poseer la información necesaria sobre el futuro, deciden correctamente sobre el consumo y la inversión (Kaletsky, 2009; Greenspan, 1998), pues los mercados dan las señales fiables para la asignación de factores de acuerdo a la optimalidad paretiana. La HME aplicada a los mercados financieros significó la desregulación de los hedge funds y los derivativos (Thaler, 2009) y es raíz del crecimiento del рIв intensivo en energía y otros recursos naturales, la contaminación y el cambio climático.

En la HME el futuro está predeterminado, se revela en la información que genera el mercado y es integrable en los modelos de equilibrio general. Esta predeterminación elimina la constante interacción reflexiva entre los agentes que intervienen en el mercado (Davidson, 2012; Soros, 1998) e ignora los efectos de esa comunicación "[...] lo que la gente piense hoy del mercado puede afectar y alterar su evolución futura" (Soros, 1998: p. 45. Traducción propia). Otros críticos ortodoxos (Akerlof \& Shiller, 2009; Shiller, 2003) señalan que la HME es un error notable, pues los agentes económicos no son perfectamente racionales, sino que suelen tener momentos de optimismo conducentes a exuberancias irracionales seguidas de pesimismo y retiro del mercado, y actúan en tropel, dado que optimismo y pánico son contagiosos. En 2008, Greenspan señaló ante el Congreso esta hipótesis como la causa de la crisis, lo que ratifican Akerlof \& Shiller (2015). El yerro de Greenspan y Bernanke, gestores de políticas públicas de impacto mundial, es haber rechazado incluso la posibilidad de una burbuja inmobiliaria y afirmar que, de existir, no explotaría pues el mercado la desinflaría oportunamente.

Por último, se cuestiona el óptimo de Pareto. Aun en el paradigma neoclásico han emergido cuestionamientos sobre el funcionamiento de los mercados y la existencia de múltiples equilibrios de precaria estabilidad. Concebir el cambio económico como Pareto-neutral (y sus diversas variantes) implica que globalmente la sociedad gana, que al menos alguien está mejor y nadie empeora - resultado plausible solo mediante compensaciones-. Esta propuesta depende de, y acepta como eficientes, las dotaciones iniciales de recursos e impide la redistribución al considerar el punto inicial óptimo y asignar valor superior a la efi-

9 Hipótesis propuesta por Fama (1970) y criticada, entre otros, por Bernstein \& Bernstein (1996), Fox (2009), Roubini (2006), Shiller (2003) y Thaler (2009). 
ciencia sobre la equidad. Su aplicación como argumento es discrecional, teórica y cargada de valor: cuando se analizaron las reformas estructurales se presentó el cambio como el óptimo social y a los grupos que no lo defendían como rentistas del "viejo régimen" que cambiarían de opinión con el crecimiento de los costos de sostener su posición (Alesina, Ardagna \& Trebbi, 2006). Pero ni el cambio ni sus resultados eran óptimos paretianos toda vez que alguien perdía en el proceso y toleraba los resultados solo por la imposibilidad de compensar a los futuros ganadores los costos de mantener el statu quo. Refuerzan este argumento escritos sobre la creciente concentración de la riqueza en el mundo, mismos que cuestionan la optimalidad paretiana de los equilibrios. En efecto, desde fines de los setenta, un minúsculo grupo acumuló ganancias siderales mientras el resto perdía, proceso que alerta sobre los riesgos de la estabilidad de la economía mundial de continuar el rumbo seguido (Piketty, 2014; Stiglitz, 2012). Al estallar la crisis se arbitraron recursos para rescatar los bancos, dejando miles de familias sin empleo y sin hogar.

\section{No obstante sus falencias, los paradigmas dominantes se fortalecen}

\section{La reproducción de los paradigmas}

¿Con tantos errores de predicción y tan difíciles de avalar sus supuestos, qué sostiene la supremacía de la escuela neoclásica? Numerosas y de diversa índole son las causas de la resistencia al cambio que ayuden a entender por qué la crisis de 2008 no ha generado los cambios esperados en la teoría y en las políticas económicas y parecería que se han fortalecido la ortodoxia, la austeridad y la maximización de ganancias financieras. Unas razones tienen que ver con el objeto mismo de la economía, esto es, sus vínculos con el poder político y económico; otras con la estructura de la comunidad de economistas (incluidos las universidades y centros de investigación) y con las normas de legitimación de las ideas y de transición del conocimiento, el contenido de los currículos y de los manuales de macroeconomía.

Con la crisis, el consenso neoclásico se agrietó y emergieron recomendaciones neokeynesianas que utilizaron los hacedores de política, urgidos por soluciones, pero carentes de argumentos neoclásicos para legitimar la austeridad (Krugman, Alan Blinder, Joseph Stiglitz, Olivier Blanchard, Greg Mankiw, Dominique Strauss). Se aceptó la necesidad de intervenir en el mercado y la magnitud de esa intervención y se instrumentaron acciones para paliar la crisis en Estados Unidos y Europa. Hacia 2010, liderada por el FMI y el Banco Central Europeo, la hegemonía neoclásica se restituía e imponía la austeridad,

A. Puyana | Crisis económica y crisis de la teoría económica. Notas para el debate Perfiles Latinoamericanos, 26(51) | FLACso México | pp. 351-378 | DoI: 10.18504/pl2651-014-2018 
según algunos autores (Blyth, 2013: 208-211), por cinco razones: a) las crisis estallan, los paradigmas cambian lentamente y la acumulación de eventos para síntesis teóricas es un proceso de largo plazo; $b$ ) las transformaciones son graduales y pocas veces absolutas; $c$ ) el divorcio no costoso entre las ideas discutidas en la academia y la realidad; las crisis impactan el mundo real pero no tocan los salones de clase, se toman como experimentos para fortalecer los supuestos; $d$ ) la renuencia de las instituciones a incorporar información que mine su autoridad, y $f$ ) el costo de recrear esa autoridad una vez erosionada; asuntos de interés político cuestionan las posibilidades en corto plazo de un cambio de paradigma en la teoría macroeconómica y su trasmisión a las políticas (Przeworski, 2015).

En macroeconomía, la competencia de ideas no siempre termina con el triunfo de las más sólidas ya que al ser tan compleja es necesariamente especulativa y, por lo tanto, casi imposible de saber a priori cuáles son las ideas más sólidas Collander (2010). De la complejidad se deriva que la comunidad macroeconómica es pequeña, organizada oligopólicamente y con margen para alentar la competencia eficaz sobre modelos e ideas alternativas. Por la conexión de la economía y la política, si un modelo racionaliza y avala propuestas o propósitos políticos, es presentado y prematuramente legitimado como el "modelo" y se clausura la consideración de otros.

Las experiencias del sistema de asignación de fondos para la investigación del Reino Unido y los Estados Unidos sugieren que la explicación estaría en los mecanismos de reproducción interna del sistema y el actuar de los académicos de esta corriente, insertos en redes que controlan los comités de evaluación de proyectos, las revistas, los departamentos y privilegian investigaciones afines a sus convicciones (Gillies, 2012; Fullbrook, 2010). Cómo se difunden y legitiman las ideas y se llega a consensos en teoría económica "tiene más que ver con las relaciones de poder que con la investigación desinteresada y revela la relación entre las ideas y el poder" (Palacios, 2005: p. 41). Por la necesidad de fondos para la investigación, los economistas acomodan sus intereses y prácticas investigativas a las preferencias, temas y objetivos de las instituciones que financian la investigación (Collander, 2010).

Al aceptar el Premio Nobel, Stigler (1982a) exploró la sociología de los economistas como actores de poder y los responsabilizó del estancamiento del conocimiento económico debido a que abandonan la comprobación de sus teorías con los hechos reales. Así, "los buenos economistas, no son aquellos que están en lo correcto, sino los que influyen en la profesión en conjunto, para lo cual y, dado que es más difícil vender nuevas ideas que nuevos productos, aplican las técnicas de persuasión del vendedor callejero: 'repetición, afirmaciones exageradas, y énfasis desproporcionados' y devienen, de estudiosos y teóricos, 
en predicadores" (Stigler, 1982b: p. 5). ${ }^{10}$ De ese entramado de la economía con el poder político y económico ha surgido una arquitectura de la profesión, cuyo núcleo es el sistema académico de los Estados Unidos centrado en las redes universitarias estadounidenses altamente jerarquizadas, las cuales aglutinan la mayor parte de los referentes mundiales: ganadores de premios Nobel, funcionarios de organismos internacionales, renombrados académicos y dominan el escenario de la economía, como explaya y generaliza Fullbrook (2010).

\section{La transmisión del conocimiento y reproducción de los paradigmas}

Entre los causales de la resistencia al cambio en la comunidad económica, es necesario considerar el papel de las facultades de economía en su calidad de transmisoras del conocimiento y forjadoras de profesionales que moldearán las relaciones económicas y sociales. Según Keynes, la sociedad requiere buenos economistas, pero "[...] economistas buenos, siquiera competentes, son aves raras [...] [El economista] debe ser matemático, historiador, filósofo, estadista [...] Ningún aspecto de la naturaleza del hombre o instituciones puede estar fuera de su reflexión” (Keynes, 1924: pp. 311-372).

Este ideal se perdió. El predominio de la econometría sobre la teoría económica y desviaciones en su enseñanza aislaron la teoría económica de la práctica y la transmisión del pensamiento, zanja que despertó inquietudes años ha. En 1988, la American Economic Association formó una comisión para valorar los programas de posgrado en teoría económica de las universidades estadounidenses y el tipo de doctores egresados. Esa comisión constató la mutación de la teoría económica en rama de las matemáticas aplicadas, desvinculada del mundo, los eventos y las instituciones reales cuyos programas de posgrado "producen generaciones de economistas, idiot savants, duchos en técnicas, pero inocentes de los hechos económicos" (Krueger, 1991: p. 3. Traducción propia). Las falencias: no enseñar historia, filosofía, geografía, instituciones y teoría económica, no leer los clásicos. En reacción a la predominancia de la economía neoclásica de la Royal Economic Society, en 1993, nació en Inglaterra e Irlanda la Confederación Internacional de Asociaciones por el Pluralismo en Economía (ICAPE, siglas en inglés), ${ }^{11}$ para procurar la diversidad de enfoques teóricos y métodos analíticos desde visiones alternativas. En septiembre de 2000, estudiantes de

10 Para un revelador análisis de los puntos de Stigler aplicado al BM, véase Moretti, F. \& Preste, D. (2015, mayo-junio). Jerga bancaria. El lenguaje empleado en los informes del Banco Mundial. New Left Review, (99), 99-107.

11 Véase http://hetecon.net

A. Puyana | Crisis económica y crisis de la teoría económica. Notas para el debate Perfiles Latinoamericanos, 26(51) | FLACso México | pp. 351-378 | DoI: 10.18504/pl2651-014-2018 
economía de la École Normale Supérieure de Francia protestaron contra la excesiva formalización matemática en la enseñanza, no por rechazo a las matemáticas, sino por entronizar, a espaldas de la realidad, el modelaje como el fin de la teoría y como el objeto del estudio del instrumento (los modelos) en lugar del tema (la economía). Invocaron el retorno al pluralismo que considere la realidad. Movimientos similares surgieron en otras latitudes alentando movimientos sociales de rechazo al "ajuste" en toda Europa y en los Estados Unidos, el cual intensifica la desigualdad a tal grado que preocupa a los organismos multilaterales y es objeto de reflexión teórica. ${ }^{12}$ La conformación de varias agrupaciones que, como Rethinking Economics, Post-Autistic Economics o Real World Economics Association, buscan cambios en la enseñanza de la economía, sugiere que la inconformidad de estudiantes y profesores continúa ${ }^{13}$ y se expresa inclusive en la no asistencia a ciertas clases y abandonando del salón en presencia del profesor. ${ }^{14}$ No se ha avanzado en la ruta de cambiar los currículos universitarios, no al menos en los Estados Unidos y el Reino Unido. Si hubo cambios, fue intensificando los defectos señalados. Wren-Lewis (2012) lamenta que brillantes estudiantes de economía no investigan problemas de la vida real, por no ser claros los supuestos microeconómicos. El 5 de mayo de 2014 nació la Iniciativa Internacional de Estudiantes por el Pluralismo en Economía (ISIPE, siglas en inglés), con estudiantes y académicos de treinta países, ${ }^{15}$ para discutir la enseñanza de la economía, pues la universidad moldea "las próximas generaciones de políticos y forma a la sociedad en que vivimos” (ISIPE, 2015). Reclama, como las iniciativas anteriores, que las aulas sean foros de debate plural de la teoría y los métodos, que "renueve la disciplina y aporte soluciones a los problemas sociales" (ISIPE, 2015). ${ }^{16}$ Pide ampliar el rango de escuelas y métodos estudiados, incorporando perspectivas de otras disciplinas, el rol del Estado, la inserción en la economía global y las relaciones de poder entre y en los países (Gambina, 2010). El Banco de Inglaterra y la Royal Economic So-

12 Robert B. Reich (2012), profesor de Berkley, documenta que el "capitalismo moderno" consolidado en las tres últimas décadas, al concentrar la riqueza, erosiona sus propias fuentes de crecimiento y mina la democracia. La OECD (2011) lamenta la concentración del ingreso por sus daños a la cohesión social y al sistema económico y político.

13 La red de profesores y estudiantes de escuelas de economía de todo el mundo desarrollado y en desarrollo, los cuales aprobaron el "Manifesto: A Direction for the Reform of Economics" (LSE, Harvard, Manchester, Oxford, Brasil, Argentina).

14 En 2011, los estudiantes de la Universidad de Harvard abandonaron la clase de Gregory Mankiw y publicaron una carta abierta explicando esa decisión. Recuperado de http://harvardpolitics.com/harvard/ an-open-letter-to-greg-mankiw/

15 En 2015, isipe cuenta con 82 grupos asociados y declaró el 5 de mayo como el día de acción global por el pluralismo en economía.

16 Disponible en http://www.isipe.net/open-letter 
ciety convocaron en 2011/2012 a economistas académicos y en altos cargos públicos y a hombres de negocios a discutir la crisis de la ciencia económica y de su enseñanza. Sus discusiones señalan cómo el exclusivismo curricular y la falta de relación entre los modelos teóricos de equilibrio general y la vida real genera profesionales sin capacidad de analizar los eventos corrientes y sus efectos, por ejemplo, las crisis, las burbujas, el pánico, los efectos de la crisis financiera, el desempleo, la desigualdad o el cambio climático (Coyle, 2012b; Royal Economic Society, 2015). Estas recomendaciones coinciden con las de Coyle (2012a, 2013) y Gilles (2012) a partir del estudio de los sistemas académicos de Reino Unido y Estados Unidos, con las propuestas de organización académica de Garnett (2011) y Mearman (2011) y de académicos establecidos como Krugman, Stiglitz o Thaler.

En ninguna otra disciplina se ha conformado una protesta tan global, organizada y amplia, pero el cambio no es evidente y cabe preguntar qué hacer para quebrar la inercia educativa y dar respuesta a las demandas de dar cabida a las diversas vertientes del pensamiento y de reformar la enseñanza de la economía, revitalizadas a raíz de la crisis de 2008 y expresadas en propuestas de catedráticos, y de expertos del mundo de los negocios y del gobierno.

Entre los planteamientos más comprensivos y de mayor alcance teórico y metodológico resultaron los de la Conferencia de la Real World Economics Association en 2013 y recogidos en Madi \& Reardon (2015). Las líneas siguientes recogen los aportes de la conferencia más relacionados con la problemática desarrollada en este ensayo. Sintetizamos los aportes de la conferencia sobre el currículo en economía en lo referente a qué se enseña y sus limitaciones epistemológicas y cómo se enseña y cómo se transmite el conocimiento teórico y empírico.

Se enseña exclusivamente economía neoclásica, en vacío histórico y político y como la única teoría válida "científica" en virtud de su sistematización matemática en modelos de equilibrio, cada vez más complejos, que son no solo abstractos, como toda síntesis teórica lo es, sino que falsifican la realidad e impiden su conocimiento. Lars (2015) apoya esta afirmación con tres supuestos neoclásicos: a) rigidez de los salarios, $b$ ) la ley de la oferta y $c$ ) la demanda y las preferencias reveladas que son más restrictivos que la variables generalmente usadas en la economía neoclásica mismas que no se pueden hacer realistas con adiciones marginales. Son críticas a la teoría que coinciden con lo expuesto en la tercera sección de este ensayo. Madi \& Readon (2015) citan a Roncaglia quien censura la omisión de la historia económica y de la historia del pensamiento económico, materias que deberían ser parte nuclear de los currículos, al evidenciar la naturaleza cambiante de los problemas económicos y la existencia de paradigmas. Los principios neoclásicos y sus derivaciones apuntan que la macroeconomía neoclásica y

A. Puyana | Crisis económica y crisis de la teoría económica. Notas para el debate Perfiles Latinoamericanos, 26(51) | FLACso México | pp. 351-378 | DoI: 10.18504/pl2651-014-2018 
sus instituciones impiden la comprensión de los problemas reales y la complejidad del actuar humano, con gustos y preferencias cambiantes, en un entorno social complejo (Reardon, 2015), al impedir estudiar problemas económicos frecuentes (el desempleo) y nuevos como la desigualdad (la más perniciosa idea de la economía, según Lucas (2003), el cambio climático y las crisis financieras. La abstracción del mercado laboral solo permite el desempleo voluntario y las preferencias se consideran estáticas y únicas. En este tenor, se aconseja remozar los currículos con los temas señalados en las secciones 3 y 4 del libro de Madi y Reardon (2015), que analiza las finanzas internacionales, las empresas multinacionales, la política económica y, trascendental, los aspectos políticos y éticos del mercado, en el sentido de Hermann (2015). Esta enseñanza corresponde a un sistema cerrado, no pluralista, deductivo y excluyente que, para Dow (2015), es antidemocrático y antipedagógico, contrario a lo que supone un sistema educativo abierto. A partir de este diagnóstico, surgieron propuestas de reformar el currículo para los estudios de pre y posgrado, entre los cuales sobresale, además del pluralismo y la introducción de la historia, posponer el estudio de las teorías económicas para semestres avanzados, luego de estudiar humanidades, filosofía e inclusive literatura universal (Balzac, Mann, Dickens, entre otros).

\section{Reflexiones finales}

La crisis financiera global siembra dudas sobre la capacidad del mercado para desatar crecimiento sostenible, reducir la desigualdad y la pobreza, extender los derechos ciudadanos universales y propiciar el uso racional de los factores, el humano, en primer lugar. Cuestionó los efectos democratizadores del mercado y la solidez de la macroeconomía hincada en los supuestos de la teoría neoclásica y basada en fundamentos microeconómicos y la naturaleza de las políticas soportadas en estos principios. Reanimó el debate sobre las credenciales de la teoría económica como ciencia exacta políticamente neutral y con capacidad de predecir. Quizás un efecto "positivo" de la crisis sea el retorno de la economía al campo de las ciencias sociales, regreso particularmente necesario para la macroeconomía, en la cual el tenue lindero entre la teoría económica y el ejercicio político se difumina. También la apertura a nuevas corrientes que responden a los cambios de la economía mundial, como la economía de la complejidad, la economía evolucionista y la economía de redes que en esencia se distancian de los presupuestos neoclásicos y los enriquecen.

Abundan las dudas sobre la posibilidad de cambios de paradigma en el pensamiento económico dominante y de una salida suave de la crisis. Al mediar 2016, las economías desarrolladas no recorren aún la senda del crecimiento y las 
que se encuentran en desarrollo tropiezan. La campaña electoral en los Estados Unidos y el Brexit, entre otros eventos políticos, avivan temores de que reculen los avances en liberalización económica y retorne el nacionalismo. Entretanto, no se desmontan las medidas de austeridad para resolver la crisis y avanza el desmonte del Estado de bienestar. El trueque de derechos ciudadanos por facilidades de crédito para el consumo extiende la crisis en el tiempo, la recesión aún no cede y se traslada a las economías periféricas.

Las críticas al paradigma dominante sostenidas por décadas pueden dar luz a un renovado pluralismo teórico con efectos democratizadores.

El debate sobre la crisis en la teoría económica se extendió a la naturaleza y estructura de la comunidad de los economistas y develó su carácter de gremio cerrado, no pluralista, y los mecanismos de control de la investigación y la transmisión de conocimientos retardan el cambio. La enseñanza refleja estas falencias en el contenido curricular y el método pedagógico, el cual reproduce el exclusivismo al enseńar solo una escuela de pensamiento, carente de sentido histórico, desvinculada de los problemas reales. Las iniciativas de reforma de la enseñanza reiteran que se enseñe a los alumnos primero a pensar e investigar críticamente los problemas económicos reales, en su contexto histórico y político, y en segundo término, en el uso de herramientas analíticas, matemáticas y de todo tipo.

\section{Referencias}

Akerlof, G. A. \& Shiller, R. J. (2009). Animal Spirits: How Human Psychology Drives the Economy, and Why It Matters for Global Capitalism. Princeton: Princeton University Press.

Akerlof, G. A. y. Shiller, R. J. (2015). Phishing for Phools: The Economics of Manipulation and Deception. Nueva York: Princeton University Press.

Alesina, A., Ardagna, S. \& Trebbi, F. (2006). Who Adjusts and When? On the Political Economy of Reforms. NBER Working Paper, núm. 12049. Washington: National Bureau of Economic Research (NBER).

Banco Mundial (вм) (2016, enero). Global Economic Prospects. Enero. Recuperado el 18 de septiembre de 2017, de http://www.worldbank.org/en/publication/global-economic-prospects

Banco Mundial (2005). Concept Note: Rethinking Social Policy.

Becker, G. S. (1996). Accounting for Tastes. Cambridge: Harvard University Press.

Bernanke, B. (2004). Essays on the Great Depression. Princeton: Princeton University Press. 
Bernstein, P. \& Bernstein, P. L. (1996). Against the Gods: The Remarkable Story of Risk (pp. 12691275). Nueva York: Wiley.

Blagrave, P. et al. (2015). Where are We Headed? Perspectives on Potential Output. En World Economic Outlook (weO). Uneven Growth: Short-and Long-Terms Factors. Washington: International Monetary Fund.

Blyth, M. (2013). Paradigms and Paradox: The Politics of Economic Ideas in Two Moments of Crisis. Governance: An International Journal of Policy, Administration, and Institutions, 26(2), 197-215.

Collander, D. (2010). Testimony of David Collander, submitted to the Congress of the United States, House Science and Technology Committee, presentado el 20 de julio de 2010 (pp. 39-44). Recuperado el 20 de abril del 2016, de https://www.gpo.gov/fdsys/pkg/ CHRG-111hhrg57604/pdf/CHRG-111hhrg57604.pdf

Coyle, D. (2012a). Do Economic Crises Reflect Crises in Economics? Economic Thought, 1(1).

Coyle, D. (2012b). On the Public Responsibilities of the Economist. Conferencia de The Tanner Lectures on Human Values dictada en Brasenose College, Oxford, 18 y 19 de mayo.

Coyle, D. (2013). The State of Economics and the Education of Economists. World Economics Association (wEA). Conferences No. 2. The Economics Curriculum: Towards a Radical Reformation, 3 de mayo-14 de junio.

Davidson, P. (2012). Is Economics a Science? Should Economics be Rigorous? Real-world Economics Review, (59), 58-66.

Davies, G. (2015, 31 de mayo). Has the Rethinking of Macroeconomic Policy Been Successful? Financial Times. Recuperado el 31 de mayo de 2015, de https://www.ft.com/content/ 5610de8e-8732-3529-bfa9-7fe2bfaa0500

Dow, S. (2013). Teaching Open-system Economics. En Madi, M. A. \& Reardon, J. (Eds.) (2015). The Economics Curriculum: Towards a Radical Reformulation, World Economics Association (WEA). Conferences núm. 2, 3 de mayo-14 de junio de 2013.

Fama, E. (1970). Efficient Capital Markets: a Review of Theory and Empirical Work. Journal of Finance, 25(2), 383-417.

Farmer, R. E. A. (2009). Fiscal Policy Can Reduce Unemployment: But There is a Better Alternative. CEPR Discussion Paper No. DP7526. Centre for Economic Policy Research (CEPR) y National Bureau of Economic Research (NBER). 
Farrell, H. \& Quiggin, J. (2012). Consensus, Dissensus and Economic Ideas: The Rise and Fall of Keynesianism During the Economic Crisis. RSMG Working Paper Series No. P11_2. Brisbane: The University of Queensland.

Financial Crisis Inquiry Commission (FCIC). (2011). The Financial Crisis Inquiry Report. Washington: Gobierno de Estados Unidos.

Fondo Monetario Internacional (FMI). (2014). World Economic Outlook. October 2014. Legacies, Clouds, Uncertainties. Washington: IMF.

Fondo Monetario Internacional (FMI). (2015). World Economic Outlook April 2015. Uneven Growth: Short- and Long-terms Factors. Washington: IMF.

Foyle, D. K. (2004). The Strange History of the Economic Agent. New School Economic Review, 1(1).

Fox, J. (2009). The Myth of the Rational Market: A History of Risk, Reward, and Delusion on Wall Street. Nueva York: HarperBusiness.

Francesco, S. (2015). L'histoire (faussement) naïve des modèles DSGE. Documento de trabajo del Centre d'Économie de la Sorbonne (CES).

Frank, M. F. (2015). 2013 Annual Report of Martin Capital Management. Recuperado el 20 de febrero de 2016, de https://www.gurufocus.com/news/248509/martin-capital -management-2013-annual-report

Fullbrook, E. (2013). New Paradigm Economics versus Old Paradigm Economics. Real-World Economics Review, (66). Recuperado el 10 de abril de 2015, de http://www.paecon.net/ PAEReview/

Fullbrook, E. (2010). How to Bring Economics into the 3rd Millennium by 2020, Real-world Economics Review, 54.

Galbraith, J. K. (2010). The Affluent Society \& Other Writings, 1952-1967: American Capitalism. The Great Crash, 1929. The Affluent Society. The New Industrial State. Nueva York: Penguin Group USA.

Galbraith, J. K. (1974). El poder y el economista útil. Trimestre Económico, 41(161-1), 231-247.

Gambina, J. (2010). La crisis de la economía mundial y los desafíos para el pensamiento crítico. En Gambina, J. (Coord.). La crisis capitalista y sus alternativas (pp. 77-92). Buenos Aires: Clacso. 
Garnett, R. (2011). Pluralism, Academic Freedom, and Heterodox Economics, Review of Radical Political Economics, 43(4), 562-572.

Gillies, D. (2012). Economics and Research Assessment Systems, Economic Thought, 1(1), $23-47$.

Greenspan, A. (1998). Testimony of Chairman Alan Greenspan.The Regulation of OTC Derivative. Comité sobre Servicios Bancarios y Financieros del Congreso de Estados Unidos, testimonio presentado el 24 de julio.

Harvey, D. (2007). Breve historia del neoliberalismo. Madrid: Akal.

Hayek, F. (1990 [1944]). Camino de servidumbre. Madrid: Alianza.

Hermann, A. (2013). Market, Socialism and Democracy in an Interdisciplinary Perspective. En Madi, M. A. \& Reardon, J. (Eds.) (2015). The Economics Curriculum: Towards a Radical Reformulation, World Economics Association (wEA). Conferences núm. 2, 3 de mayo-14 de junio de 2013.

ISIPE (2015). An international student call for pluralism in economics. Recuperado de http:// www.isipe.net/open-letter

Jomo, K. S. (Ed.). (2005). The Pioneers of Development Economics. Great Economists on Development. Nueva York, Londres: Zed Books.

Kaletsky, A. (2009). Goodbye, Homo Economicus. Prospect Magazine. Recuperado el 22 de agosto de 2015, de https://www.prospectmagazine.co.uk/magazine/goodbyehomoeconomicus

Kay, J. (2014). The Map Is not the Territory: An Essay on the State of Economics. Revista de Economía Institucional, 16(30), 13-24.

Keynes, J. M. (1924). Alfred Marshall, 1842-1924. The Economic Journal, 34(135), 311-372.

Korinek, A. (2015). Matching the Moment, But Missing the Point? Institute for New Economic thinking (INET). Recuperado el 25 de marzo de 2015, de https://www.ineteconomics.org/ perspectives/blog/matching-the-moment-but-missing-the-point

Krueger, A. (1991). Report of the Commission on Graduate Education in Economics. Journal of Economic Literature, 1035-1053.

Krugman, P. (2011). The Profession and the Crisis. Eastern Economic Journal, 37(3), 307-312. 
Krugman, P. (2009a). A Dark Age of Macroeconomics (Wonkish). The New York Times Blog, 27 de enero. Recuperado el 29 de junio de 2009, de https://krugman.blogs.nytimes .com/2009/01/27/a-dark-age-of-macroeconomics-wonkish/?mcubz=0\&_r=0

Krugman, P. (2009b). How Did Economists Get It So Wrong? The New York Times Magazine, Septiembre 2 de 2009. Recuperado el 18 de septiembre de 2017, de http://www.nytimes .com/2009/09/06/magazine/06Economic-t.html?mcubz=0

Krugman, P. (1999). The Fall And Rise of Development Economics. Recuperado el 18 de marzo de 2015, de http://web.mit.edu/krugman/www/dishpan.html

Lagarde, C. (2015). Global Growth will be Disappointing in 2016, Reuters. Recuperado de www .reuters.com/article/us-imf-lagarde-idUSKBNOUD0JB20151230.

Luigi Buttiglione, Philip R. Lane, Lucrezia Reichlin \& Vincent Reinhart (2014). Deleveraging? What Deleveraging? The 16th Geneva Report on the World Economy. Reino Unido: Centro Internacional de Estudios Monetarios y Bancarios (ICMBs). Recuperado el 18 de septiembre de 2017, de http://cepr.org/sites/default/files/news/Geneva16_0.pdf

Lipton, M. (1992). The State-market Dilemma, Civil Society, and Structural Adjustment. Any Cross-commonwealth Lessons? The Round Table, 80(317), 21-31.

Lucas, R. (2003). Macroeconomic Priorities. American Economic Review, 93(1), 1-14.

Lucas, R. \& Sargent, T. (1979). After Keynesian Macroeconomics. Federal Reserve Bank of Minneapolis Quarterly Review, 3(2), 1-16.

Lynn, S. (2003). Economic Development: Theory and Practice for a Divided World. Nueva Jersey: Prentice Hall.

Madi, M. A. \& Reardon J. (Eds.). (2015). The Economics Curriculum: Towards a Radical Reformulation, World Economics Association (WEA). Conferences No. 2, 2013, The Economics Curriculum: Towards a Radical Reformation, 3 de mayo-14 de junio de 2013.

Marchionatti, R. \& Sella, L. (2015). Is Neo-Walrasian Macroeconomics a Dead End? CESMeP Working Paper 21/2015. Torino: Centro di Studi sulla Storia e I Metodi dell'Economia Politica "Claudio Napoleoni".

Mearman, A. (2011). Pluralism, Heterodoxy, and the Rhetoric of Distinction, Review of Radical Political Economics, 43(4), 552-561.

Minsky, H. (2008 [1986]). Stabilizing an Unstable Economy. Nueva York: McGraw-Hill Professional. 
Moretti, F. \& Preste, D. (2015). Jerga bancaria. El lenguaje empleado en los informes del Banco Mundial, New Left Review, 92, 81-107.

Organización para la Cooperación y el Desarrollo Económicos (OCDE). (2016). World Economic Outlook, junio.

Organisation for Economic Co-operation and Development (oeCD). (2011). Divided We Stand: Why Inequality Keeps Raising. París: OECD.

Palacios, M. A. (2005). Knowledge is Power: The Case of Colombian Economists. En Fitzgerald, V. \& Thorp, R. (Eds.). Economic Doctrines in Latin America: Embedding and Evolution. Oxford: Palgrave.

Piketty, T. (2014). Capital in the Twenty-First Century. Cambridge: Harvard University Press.

Prins, N. (2015). Hillary Clinton's Chronic Caution on the Big Banks: Her Economic Agenda is Missing the One Sure-fire Way to Rein in their Runaway Power", New York Daily News, 14 de julio de 2015.

Przeworski, A. (2015). Desigualdad económica, desigualdad política y redistribución. Conferencia dictada en el 40 Aniversario de la Flacso México, 28 de octubre. [Video]. Recuperado de https://www.youtube.com/watch?v=wAdvEzEOhbg

Quiggin, J. (2010). Zombie Economics: How Dead Ideas Still Walk Among Us. Princeton: Princeton University Press.

Reardon, J. (2013). A Radical Reformation of Economics Education: Educating Real World Economists. En Madi, M. A. \& Reardon, J. (Eds.) (2015). The Economics Curriculum: Towards a Radical Reformulation, World Economics Association (WEA). Conferences núm. 2, 3 de mayo-14 de junio de 2013.

Reich, R. B. (2012). Beyond Outrage: What Has Gone Wrong With Our Economy and Our Democracy, and How to Fix It. Nueva York: Vintage Books.

RDMP (2009). Her Majesty the Queen... Revue du MAUSS.

Rogoff, K. (2002). Dornbusch's Overshooting Model After Twenty-Five Years. IMF Working Paper No. 02/39. Washington, EEUU: International Monetary Fund.

Roncaglia, A. (2013). Should the History of Economic Thought be Included in Undergraduate Curricula? En Madi, M. A. \& Reardon, J. (Eds.) (2015). The Economics Curriculum: Towards a Radical Reformulation, World Economics Association (WEA). Conferences núm. 2, 3 de mayo-14 de junio de 2013. 
Rosenthal, G. (2010). La crisis financiera y económica de 2008 y su repercusión en el pensamiento económico. Revista de la CEPAL, (100), 29-39.

Roubini, N.(2006). Why Central Banks Should Burst Bubbles. International Finance, 9(1), 87-107.

Royal Economic Society (2015). Economics as a Pluralist Liberal Education. RES Newsletter, 168.

Samuelson, P. (1970). Is Economics a Science? Entrevista. Recuperado el 20 de diciembre de 2015, de https:/www.ubs.com/microsites/nobel-perspectives/en/paul-samuelson.html

Samuelson, P. (1969). Classical and Neoclassical Theory. En Clower, R. W. (Ed.). Monetary Theory (pp. 104-105). Londres: Penguin Books.

Scott, M. (1991). A New View of Economic Growth. Oxford: Oxford, University Press.

Shiller, R. (2003). From Efficient Markets Theory to Behavioral Finance. Journal of Economics Perspective, 17(1), 83-104.

Silim, A. (2012). What is New Economic Thinking? En Dolphin, T. \& Nash, D. (Eds.). Complex New World. Translating New Economic Thinking in to Public Policy (pp. 18-27). Londres: Institute for Public Policy Research (IPPR).

Soros, G. (1998). The Crisis of Global Capitalism. Boston: Little, Brown and Company.

Stigler, G. (1982a). The Process and Progress in Economics. Conferencia de aceptación del Premio Nobel. Recuperado el 18 de octubre de 2015, de http://www.journals.uchicago.edu/ doi/abs/10.1086/261165.

Stigler, G. (1982b). The Economist as Preacher and Other Essays. Chicago: University of Chicago Press.

Stiglitz, J. (2012). The Price of Inequality: How Today's Divided Society Endangers Our Future. Nueva York: W. W. Norton \& Company.

Stiglitz, J. (2009). The Anatomy of a Murder: Who Killed America's Economy? Critical Review, 21(2-3).

Stiglitz, J. (1991). The Invisible Hand and Modern Welfare Economics. Working Paper No. 3641. Washington: National Bureau of Economic Research (NBER).

Stock, J. H. \& Watson, M. (2002). Has the Business Cycle Changed and Why? NBER Macroeconomics Annual. Recuperado el 10 de diciembre de 2010, de http://www.nber.org/papers/w9127 
Syll, L. P. (2013). Economics textbooks-anomalies and transmogrification of truth. En Madi, M. A. \& Reardon, J. (Eds.) (2015). The Economics Curriculum: Towards a Radical Reformulation, World Economics Association (WEA). Conferences núm. 2, 3 de mayo-14 de junio de 2013.

Summers, L. (2014). U.S. Economic Prospects: Secular Stagnation, Hysteresis, and the Zero Lower Bound. Business Economics, 49(2), 65-73.

Summers, L. (2013). Presentación del FMI en la 14 Conferencia Anual de Investigaciones del FMI en honor de Stanley Fisher, realizada el 8 de noviembre.

Thaler, R. (2009). Markets Can Be Wrong and the Price is not Always Right. Financial Times. 4 de agosto.

Udry, C.-A.(1997). Los orígenes del neoliberalismo, Viento Sur, VI(31), 89-94.

vox/cepr Policy Portal (2016), Making the Eurozone More Resilient: What is Needed Now and What Can Wait? Recuperado el 12 de diciembre de 2016, de http://voxeu.org/article/ making-eurozone-more-resilient-what-needed-now-and-what-can-wait

Wall Street Journal. (2008). Greenspan Testimony on Sources of Financial Crisis, 23 de octubre.

Woodward, D. \& Sims, A. (2006). Growth is Failing the Poor: The Unbalanced Distribution of the Benefits and Costs of Global Economic Growth. DESA Working Paper No. 20 ST/ ESA/2006/DWP/20. Nueva York: Departament Economic \& Social Affairs.

Wren-Lewis, S. (2012). Modelling What You Can See, Mainly Macro, 18 de julio. Recuperado 16 de diciembre de 2015, de https://mainlymacro.blogspot.mx/2012/07/modelling-whatyou-can-see.html

Recibido el 18 de enero de 2015. Aceptado el 13 de octubre de 2016. 